Moroccan J. Pure and Appl. Anal. (MJPAA)

Volume 1(1), 2015, Pages 1-21

ISSN: 2351-8227

RESEARCH ARTICLE

\title{
Inequalities of Hermite-Hadamard Type
}

S. S. DRAGOMIR ${ }^{1,2}$

AbStract. Some inequalities of Hermite-Hadamard type for $\lambda$-convex functions defined on convex subsets in real or complex linear spaces are given. Applications for norm inequalities are provided as well.

1991 Mathematics Subject Classification. 26D15; 25D10.

Key words and phrases. Convex functions, Integral inequalities, $\lambda$-Convex functions.

\section{Introduction}

We recall here some concepts of convexity that are well known in the literature.

Let $I$ be an interval in $\mathbb{R}$.

Definition 1.1 ([38]). We say that $f: I \rightarrow \mathbb{R}$ is a Godunova-Levin function or that $f$ belongs to the class $Q(I)$ if $f$ is non-negative and for all $x, y \in I$ and $t \in(0,1)$ we have

$$
f(t x+(1-t) y) \leq \frac{1}{t} f(x)+\frac{1}{1-t} f(y) .
$$

Received Marsh 16, 2015 - Accepted April 15, 2015.

(C)The Author(s) 2015.This article is published with open access by Sidi Mohamed Ben Abdallah University

${ }^{1}$ Mathematics, College of Engineering \& Science Victoria University,

PO Box 14428 Melbourne City, MC 8001,

Australia.

E-Mail: sever.dragomir@vu.edu.au

http: rgmia.org/dragomir

${ }^{2}$ School of Computational \& Applied Mathematics, University of the Witwatersrand, Private Bag 3, Johannesburg 2050,

South Africa. 
Some further properties of this class of functions can be found in [28], [29], [31], [44], [47] and [48]. Among others, it has been noted that nonnegative monotone and non-negative convex functions belong to this class of functions.

The above concept can be extended for functions $f: C \subseteq X \rightarrow[0, \infty)$ where $C$ is a convex subset of the real or complex linear space $X$ and inequality (1) is satisfied for any vectors $x, y \in C$ and $t \in(0,1)$. If the function $f: C \subseteq X \rightarrow \mathbb{R}$ is non-negative and convex, then is of Godunova-Levin type.

Definition 1.2 ([31]). We say that a function $f: I \rightarrow \mathbb{R}$ belongs to the class $P(I)$ if it is nonnegative and for all $x, y \in I$ and $t \in[0,1]$ we have

$$
f(t x+(1-t) y) \leq f(x)+f(y) .
$$

Obviously $Q(I)$ contains $P(I)$ and for applications it is important to note that also $P(I)$ contain all nonnegative monotone, convex and quasi convex functions, i. e. nonnegative functions satisfying

$$
f(t x+(1-t) y) \leq \max \{f(x), f(y)\}
$$

for all $x, y \in I$ and $t \in[0,1]$.

For some results on $P$-functions see [31] and [45] while for quasi convex functions, the reader can consult [30].

If $f: C \subseteq X \rightarrow[0, \infty)$, where $C$ is a convex subset of the real or complex linear space $X$, then we say that it is of $P$-type (or quasi-convex) if the inequality (2) (or (3)) holds true for $x, y \in C$ and $t \in[0,1]$.

Definition $1.3([7])$. Let $s$ be a real number, $s \in(0,1]$. A function $f$ : $[0, \infty) \rightarrow[0, \infty)$ is said to be s-convex (in the second sense) or Breckner $s$-convex if

$$
f(t x+(1-t) y) \leq t^{s} f(x)+(1-t)^{s} f(y)
$$

for all $x, y \in[0, \infty)$ and $t \in[0,1]$.

For some properties of this class of functions see [1], [2], [7], [8], [26], [27], [39], [41] and [50].

The concept of Breckner s-convexity can be similarly extended for functions defined on convex subsets of linear spaces.

It is well known that if $(X,\|\cdot\|)$ is a normed linear space, then the function $f(x)=\|x\|^{p}, p \geq 1$ is convex on $X$.

Utilising the elementary inequality $(a+b)^{s} \leq a^{s}+b^{s}$ that holds for any $a, b \geq 0$ and $s \in(0,1]$, we have for the function $g(x)=\|x\|^{s}$ that

$$
\begin{aligned}
g(t x+(1-t) y) & =\|t x+(1-t) y\|^{s} \leq(t\|x\|+(1-t)\|y\|)^{s} \\
& \leq(t\|x\|)^{s}+[(1-t)\|y\|]^{s} \\
& =t^{s} g(x)+(1-t)^{s} g(y)
\end{aligned}
$$


for any $x, y \in X$ and $t \in[0,1]$, which shows that $g$ is Breckner $s$-convex on $X$.

In order to unify the above concepts for functions of real variable, S. Varošanec introduced the concept of $h$-convex functions as follows.

Assume that $I$ and $J$ are intervals in $\mathbb{R},(0,1) \subseteq J$ and functions $h$ and $f$ are real non-negative functions defined in $J$ and $I$, respectively.

Definition $1.4([53])$. Let $h: J \rightarrow[0, \infty)$ with $h$ not identical to 0 . We say that $f: I \rightarrow[0, \infty)$ is an h-convex function if for all $x, y \in I$ we have

$$
f(t x+(1-t) y) \leq h(t) f(x)+h(1-t) f(y)
$$

for all $t \in(0,1)$.

For some results concerning this class of functions see [53], [6], [42], [51], [49] and [52].

This concept can be extended for functions defined on convex subsets of linear spaces in the same way as above by replacing the interval $I$ with the corresponding convex subset $C$ of the linear space $X$.

We can introduce now another class of functions.

Definition 1.5. We say that the function $f: C \subseteq X \rightarrow[0, \infty)$ is of $s$-Godunova-Levin type, with $s \in[0,1]$, if

$$
f(t x+(1-t) y) \leq \frac{1}{t^{s}} f(x)+\frac{1}{(1-t)^{s}} f(y),
$$

for all $t \in(0,1)$ and $x, y \in C$.

We observe that for $s=0$ we obtain the class of $P$-functions while for $s=1$ we obtain the class of Godunova-Levin. If we denote by $Q_{s}(C)$ the class of $s$-Godunova-Levin functions defined on $C$, then we obviously have

$$
P(C)=Q_{0}(C) \subseteq Q_{s_{1}}(C) \subseteq Q_{s_{2}}(C) \subseteq Q_{1}(C)=Q(C)
$$

for $0 \leq s_{1} \leq s_{2} \leq 1$

For different inequalities related to these classes of functions, see [1]-[4], [6], [9]-[37], [40]-[42] and [45]-[52].

A function $h: J \rightarrow \mathbb{R}$ is said to be supermultiplicative if

$$
h(t s) \geq h(t) h(s) \text { for any } t, s \in J .
$$

If inequality (6) is reversed, then $h$ is said to be submultiplicative. If the equality holds in (6) then $h$ is said to be a multiplicative function on $J$.

In [53] it has been noted that if $h:[0, \infty) \rightarrow[0, \infty)$ with $h(t)=$ $(x+c)^{p-1}$, then for $c=0$ the function $h$ is multiplicative. If $c \geq 1$, then for $p \in(0,1)$ the function $h$ is supermultiplicative and for $p>1$ the function is submultiplicative. 
We observe that, if $h$ and $g$ are nonnegative and supermultiplicative, so is their product. In particular, if $h$ is supermultiplicative then its product with a power function $\ell_{r}(t)=t^{r}$ is also supermultiplicative.

We can prove now the following generalization of the Hermite-Hadamard inequality for $h$-convex functions defined on convex subsets of linear spaces.

Theorem 1.1. Assume that the function $f: C \subseteq X \rightarrow[0, \infty)$ is an $h$-convex function with $h \in L[0,1]$. Let $y, x \in C$ with $y \neq x$ and assume that the mapping $[0,1] \ni t \mapsto f[(1-t) x+t y]$ is Lebesgue integrable on $[0,1]$. Then

$$
\frac{1}{2 h\left(\frac{1}{2}\right)} f\left(\frac{x+y}{2}\right) \leq \int_{0}^{1} f[(1-t) x+t y] d t<[f(x)+f(y)] \int_{0}^{1} h(t) d t .
$$

Proof. By the $h$-convexity of $f$ we have

$$
f(t x+(1-t) y) \leq h(t) f(x)+h(1-t) f(y)
$$

for any $t \in[0,1]$.

Integrating $(8)$ on $[0,1]$ over $t$, we get

$$
\int_{0}^{1} f(t x+(1-t) y) d t \leq f(x) \int_{0}^{1} h(t) d t+f(y) \int_{0}^{1} h(1-t) d t
$$

and since $\int_{0}^{1} h(t) d t=\int_{0}^{1} h(1-t) d t$, we get the second part of $(7)$.

From the $h$-convexity of $f$ we have

$$
f\left(\frac{z+w}{2}\right) \leq h\left(\frac{1}{2}\right)[f(z)+f(w)]
$$

for any $z, w \in C$.

If we take in $(9) z=t x+(1-t) y$ and $w=(1-t) x+t y$, then we get

$$
f\left(\frac{x+y}{2}\right) \leq h\left(\frac{1}{2}\right)[f(t x+(1-t) y)+f((1-t) x+t y)]
$$

for any $t \in[0,1]$.

Integrating (10) on $[0,1]$ over $t$ and taking into account that

$$
\int_{0}^{1} f(t x+(1-t) y) d t=\int_{0}^{1} f((1-t) x+t y) d t
$$

we get the first inequality in (7).

Remark 1.1. If $f: I \rightarrow[0, \infty)$ is an $h$-convex function on an interval $I$ of real numbers with $h \in L[0,1]$ and $f \in L[a, b]$ with $a, b \in I, a<b$, 
then from (7) we get the Hermite-Hadamard type inequality obtained by Sarikaya et al. in [49]

$$
\frac{1}{2 h\left(\frac{1}{2}\right)} f\left(\frac{a+b}{2}\right) \leq \int_{a}^{b} f(u) d u \leq[f(a)+f(b)] \int_{0}^{1} h(t) d t .
$$

If we write (7) for $h(t)=t$, then we get the classical Hermite-Hadamard inequality for convex functions.

If we write (7) for the case of $P$-type functions $f: C \rightarrow[0, \infty)$, i.e., $h(t)=1, t \in[0,1]$, then we get the inequality

$$
\frac{1}{2} f\left(\frac{x+y}{2}\right) \leq \int_{0}^{1} f[(1-t) x+t y] d t \leq f(x)+f(y),
$$

that has been obtained for functions of a real variable in [31].

If $f$ is Breckner $s$-convex on $C$, for $s \in(0,1)$, then by taking $h(t)=t^{s}$ in (7) we get

$$
2^{s-1} f\left(\frac{x+y}{2}\right) \leq \int_{0}^{1} f[(1-t) x+t y] d t \leq \frac{f(x)+f(y)}{s+1},
$$

that was obtained for functions of a real variable in [26].

Since the function $g(x)=\|x\|^{s}$ is Breckner $s$-convex on on the normed linear space $X, s \in(0,1)$, then for any $x, y \in X$ we have

$$
\frac{1}{2}\|x+y\|^{s} \leq \int_{0}^{1}\|(1-t) x+t y\|^{s} d t \leq \frac{\|x\|^{s}+\|x\|^{s}}{s+1} .
$$

If $f: C \rightarrow[0, \infty)$ is of $s$-Godunova-Levin type, with $s \in[0,1)$, then

$$
\frac{1}{2^{s+1}} f\left(\frac{x+y}{2}\right) \leq \int_{0}^{1} f[(1-t) x+t y] d t \leq \frac{f(x)+f(y)}{1-s} .
$$

We notice that for $s=1$ the first inequality in (14) still holds, i.e.

$$
\frac{1}{4} f\left(\frac{x+y}{2}\right) \leq \int_{0}^{1} f[(1-t) x+t y] d t .
$$

The case for functions of real variables was obtained for the first time in [31].

\section{2. $\lambda$-Convex Functions}

We start with the following definition:

Definition 2.1. Let $\lambda:[0, \infty) \rightarrow[0, \infty)$ be a function with the property that $\lambda(t)>0$ for all $t>0$. A mapping $f: C \rightarrow \mathbb{R}$ defined on convex subset $C$ of a linear space $X$ is called $\lambda$-convex on $C$ if

$$
f\left(\frac{\alpha x+\beta y}{\alpha+\beta}\right) \leq \frac{\lambda(\alpha) f(x)+\lambda(\beta) f(y)}{\lambda(\alpha+\beta)}
$$


for all $\alpha, \beta \geq 0$ with $\alpha+\beta>0$ and $x, y \in C$.

We observe that if $f: C \rightarrow \mathbb{R}$ is $\lambda$-convex on $C$, then $f$ is $h$-convex on $C$ with $h(t)=\frac{\lambda(t)}{\lambda(1)}, t \in[0,1]$.

If $f: C \rightarrow[0, \infty)$ is $h$-convex function with $h$ supermultiplicative on $[0, \infty)$, then $f$ is $\lambda$-convex with $\lambda=h$.

Indeed, if $\alpha, \beta \geq 0$ with $\alpha+\beta>0$ and $x, y \in C$ then

$$
\begin{aligned}
f\left(\frac{\alpha x+\beta y}{\alpha+\beta}\right) & \leq h\left(\frac{\alpha}{\alpha+\beta}\right) f(x)+h\left(\frac{\beta}{\alpha+\beta}\right) f(y) \\
& \leq \frac{h(\alpha) f(x)+h(\beta) f(y)}{h(\alpha+\beta)} .
\end{aligned}
$$

The following proposition contain some properties of $\lambda$-convex functions.

Proposition 2.1. Let $f: C \rightarrow \mathbb{R}$ be a $\lambda$-convex function on $C$.

(i) If $\lambda(0)>0$, then we have $f(x) \geq 0$ for all $x \in C$;

(ii) If there exists $x_{0} \in C$ so that $f\left(x_{0}\right)>0$, then

$$
\lambda(\alpha+\beta) \leq \lambda(\alpha)+\lambda(\beta)
$$

for all $\alpha, \beta>0$, i.e. the mapping $\lambda$ is subadditive on $(0, \infty)$.

(iii) If there exists $x_{0}, y_{0} \in C$ with $f\left(x_{0}\right)>0$ and $f\left(y_{0}\right)<0$, then

$$
\lambda(\alpha+\beta)=\lambda(\alpha)+\lambda(\beta)
$$

for all $\alpha, \beta>0$, i.e. the mapping $\lambda$ is additive on $(0, \infty)$.

Proof. (i) For every $\beta>0$ and $x, y \in C$ we can state

$$
f\left(\frac{0 x+\beta y}{0+\beta}\right) \leq \frac{\lambda(0) f(x)+\lambda(\beta) f(y)}{\lambda(\beta)}
$$

from where we get

$$
f(y) \leq \frac{\lambda(0)}{\lambda(\beta)} f(x)+f(y)
$$

and since $\lambda(0)>0$ we get that $f(x) \geq 0$ for all $x \in C$.

(ii) For all $\alpha, \beta>0$ we have

$$
f\left(\frac{\alpha x_{0}+\beta x_{0}}{\alpha+\beta}\right) \leq \frac{\lambda(\alpha) f\left(x_{0}\right)+\lambda(\beta) f\left(x_{0}\right)}{\lambda(\alpha+\beta)}
$$

from where we get

$$
f\left(x_{0}\right) \leq \frac{\lambda(\alpha)+\lambda(\beta)}{\lambda(\alpha+\beta)} f\left(x_{0}\right)
$$

and since $f\left(x_{0}\right)>0$, then we get that $\lambda(\alpha+\beta) \leq \lambda(\alpha)+\lambda(\beta)$ for all $\alpha, \beta>0$. 
(iii) If we write the inequality for $y_{0}$ we also have

$$
f\left(y_{0}\right) \leq \frac{\lambda(\alpha)+\lambda(\beta)}{\lambda(\alpha+\beta)} f\left(y_{0}\right)
$$

and since $f\left(y_{0}\right)<0$ we get that

$$
\lambda(\alpha+\beta) \geq \lambda(\alpha)+\lambda(\beta)
$$

for all $\alpha, \beta>0$.

We have the following result providing many examples of subadditive functions $\lambda:[0, \infty) \rightarrow[0, \infty)$.

Theorem 2.1. Let $h(z)=\sum_{n=0}^{\infty} a_{n} z^{n}$ a power series with nonnegative coefficients $a_{n} \geq 0$ for all $n \in \mathbb{N}$ and convergent on the open disk $D(0, R)$ with $R>0$ or $R=\infty$. If $r \in(0, R)$ then the function $\lambda_{r}:[0, \infty) \rightarrow[0, \infty)$ given by

$$
\lambda_{r}(t):=\ln \left[\frac{h(r)}{h(r \exp (-t))}\right]
$$

is nonnegative, increasing and subadditive on $[0, \infty)$.

Proof. We use the Čebyšev inequality for synchronous (the same monotonicity) sequences $\left(c_{i}\right)_{i \in \mathbb{N}},\left(b_{i}\right)_{i \in \mathbb{N}}$ and nonnegative weights $\left(p_{i}\right)_{i \in \mathbb{N}}$, namely

$$
\sum_{i=0}^{n} p_{i} \sum_{i=0}^{n} p_{i} c_{i} b_{i} \geq \sum_{i=0}^{n} p_{i} c_{i} \sum_{i=0}^{n} p_{i} b_{i}
$$

for any $n \in \mathbb{N}$.

Let $t, s \in(0,1)$ and define the sequences $c_{i}:=t^{i}, b_{i}:=s^{i}$. These sequences are decreasing and if we apply Čebyšev's inequality for these sequences and the weights $p_{i}:=a_{i} r^{i} \geq 0$ we get

$$
\sum_{i=0}^{n} a_{i} r^{i} \sum_{i=0}^{n} a_{i}(r t s)^{i} \geq \sum_{i=0}^{n} a_{i}(r t)^{i} \sum_{i=0}^{n} a_{i}(r s)^{i}
$$

for any $n \in \mathbb{N}$.

Since the series

$$
\sum_{i=0}^{\infty} a_{i} r^{i}, \sum_{i=0}^{\infty} a_{i}(r t s)^{i}, \sum_{i=0}^{\infty} a_{i}(r t)^{i} \text { and } \sum_{i=0}^{\infty} a_{i}(r s)^{i}
$$

are convergent, then by letting $n \rightarrow \infty$ in (19) we get

$$
h(r) h(r t s) \geq h(r t) h(r s)
$$

which can be written as

$$
\frac{h(r)}{h(r t s)} \leq \frac{h(r)}{h(r t)} \cdot \frac{h(r)}{h(r s)}
$$

for any $t, s \in(0,1)$. 
Let $\alpha, \beta \geq 0$ with $\alpha+\beta>0$. Then

$$
\begin{aligned}
\lambda_{r}(\alpha+\beta) & =\ln \left[\frac{h(r)}{h(r \exp (-\alpha-\beta))}\right]=\ln \left[\frac{h(r)}{h(r \exp (-\alpha) \exp (-\beta))}\right] \\
& =\ln \left[\frac{h(r)}{h(r \exp (-\alpha))} \cdot \frac{h(r)}{h(r \exp (-\beta))}\right] \\
& =\ln \left[\frac{h(r)}{h(r \exp (-\alpha))}\right]+\ln \left[\frac{h(r)}{h(r \exp (-\beta))}\right] \\
& =\lambda_{r}(\alpha)+\lambda_{r}(\beta) .
\end{aligned}
$$

Since $h(r) \geq h(r \exp (-t))$ for any $t \in[0, \infty)$ we deduce that $\lambda_{r}$ is nonnegative and subadditive on $[0, \infty)$.

Now, observe that $\lambda_{r}$ is differentiable on $(0, \infty)$ and

$$
\begin{aligned}
\lambda_{r}^{\prime}(t) & :=-(\ln [h(r \exp (-t))])^{\prime} \\
= & -\frac{h^{\prime}(r \exp (-t))(r \exp (-t))^{\prime}}{h(r \exp (-t))} \\
= & \frac{r \exp (-t) h^{\prime}(r \exp (-t))}{h(r \exp (-t))} \geq 0
\end{aligned}
$$

for $t \in(0, \infty)$, where

$$
h^{\prime}(z)=\sum_{n=1}^{\infty} n a_{n} z^{n-1} .
$$

This proves the monotonicity of $\lambda_{r}$.

We have the following fundamental examples of power series with positive coefficients

$$
\begin{aligned}
& h(z)=\sum_{n=0}^{\infty} z^{n}=\frac{1}{1-z}, z \in D(0,1) \\
& h(z)=\sum_{n=0}^{\infty} \frac{1}{n !} z^{n}=\exp (z) \quad z \in \mathbb{C}, \\
& h(z)=\sum_{n=0}^{\infty} \frac{1}{(2 n) !} z^{2 n}=\cosh z, z \in \mathbb{C} ; \\
& h(z)=\sum_{n=0}^{\infty} \frac{1}{(2 n+1) !} z^{2 n+1}=\sinh z, z \in \mathbb{C} ; \\
& h(z)=\sum_{n=1}^{\infty} \frac{1}{n} z^{n}=\ln \frac{1}{1-z}, z \in D(0,1) .
\end{aligned}
$$


Other important examples of functions as power series representations with positive coefficients are:

$$
\begin{aligned}
h(z) & =\sum_{n=1}^{\infty} \frac{1}{2 n-1} z^{2 n-1}=\frac{1}{2} \ln \left(\frac{1+z}{1-z}\right), \quad z \in D(0,1) \\
h(z) & =\sum_{n=0}^{\infty} \frac{\Gamma\left(n+\frac{1}{2}\right)}{\sqrt{\pi}(2 n+1) n !} z^{2 n+1}=\sin ^{-1}(z), \quad z \in D(0,1) \\
h(z) & =\sum_{n=1}^{\infty} \frac{1}{2 n-1} z^{2 n-1}=\tanh ^{-1}(z), \quad z \in D(0,1) ; \\
h(z) & ={ }_{2} F_{1}(\alpha, \beta, \gamma, z)=\sum_{n=0}^{\infty} \frac{\Gamma(n+\alpha) \Gamma(n+\beta) \Gamma(\gamma)}{n ! \Gamma(\alpha) \Gamma(\beta) \Gamma(n+\gamma)} z^{n}, \alpha, \beta, \gamma>0 \\
z & \in D(0,1) ;
\end{aligned}
$$

where $\Gamma$ is Gamma function.

Remark 2.1. Now, if we take $h(z)=\frac{1}{1-z}, z \in D(0,1)$, then

$$
\lambda_{r}(t)=\ln \left[\frac{1-r \exp (-t)}{1-r}\right]
$$

is nonnegative, increasing and subadditive on $[0, \infty)$ for any $r \in(0,1)$.

If we take $h(z)=\exp (z), z \in \mathbb{C}$, then

$$
\lambda_{r}(t)=r[1-\exp (-t)]
$$

is nonnegative, increasing and subadditive on $[0, \infty)$ for any $r>0$.

Corollary 2.1. Let $h(z)=\sum_{n=0}^{\infty} a_{n} z^{n}$ a power series with nonnegative coefficients $a_{n} \geq 0$ for all $n \in \mathbb{N}$ and convergent on the open disk $D(0, R)$ with $R>0$ or $R=\infty$ and $r \in(0, R)$. For a mapping $f: C \rightarrow \mathbb{R}$ defined on convex subset $C$ of a linear space $X$, the following statements are equivalent:

(i) The function $f$ is $\lambda_{r}$-convex with $\lambda_{r}:[0, \infty) \rightarrow[0, \infty)$,

$$
\lambda_{r}(t):=\ln \left[\frac{h(r)}{h(r \exp (-t))}\right] ;
$$

(ii) We have the inequality

$$
\begin{aligned}
& {\left[\frac{h(r)}{h(r \exp (-\alpha-\beta))}\right]^{f\left(\frac{\alpha x+\beta y}{\alpha+\beta}\right)}} \\
& \leq\left[\frac{h(r)}{h(r \exp (-\alpha))}\right]^{f(x)}\left[\frac{h(r)}{h(r \exp (-\beta))}\right]^{f(y)}
\end{aligned}
$$

for any $\alpha, \beta \geq 0$ with $\alpha+\beta>0$ and $x, y \in C$. 
(iii) We have the inequality

$$
\begin{aligned}
& \frac{[h(r \exp (-\alpha))]^{f(x)}[h(r \exp (-\beta))]^{f(y)}}{[h(r \exp (-\alpha-\beta))]^{f\left(\frac{\alpha x+\beta y}{\alpha+\beta}\right)}} \\
& \leq[h(r)]^{f(x)+f(y)-f\left(\frac{\alpha x+\beta y}{\alpha+\beta}\right)}
\end{aligned}
$$

for any $\alpha, \beta \geq 0$ with $\alpha+\beta>0$ and $x, y \in C$.

Proof. We have

$$
f\left(\frac{\alpha x+\beta y}{\alpha+\beta}\right) \lambda_{r}(\alpha+\beta) \leq \lambda_{r}(\alpha) f(x)+\lambda_{r}(\beta) f(y)
$$

for any $\alpha, \beta \geq 0$ with $\alpha+\beta>0$ and $x, y \in C$, is equivalent to

$$
\begin{aligned}
& \ln \left[\frac{h(r)}{h(r \exp (-\alpha-\beta))}\right]^{f\left(\frac{\alpha x+\beta y}{\alpha+\beta}\right)} \\
& \leq \ln \left[\frac{h(r)}{h(r \exp (-\alpha))}\right]^{f(x)}+\ln \left[\frac{h(r)}{h(r \exp (-\beta))}\right]^{f(y)} \\
& =\ln \left\{\left[\frac{h(r)}{h(r \exp (-\alpha))}\right]^{f(x)}\left[\frac{h(r)}{h(r \exp (-\beta))}\right]^{f(y)}\right\}
\end{aligned}
$$

for any $\alpha, \beta \geq 0$ with $\alpha+\beta>0$ and $x, y \in C$.

The inequality (28) is equivalent to (26) and the proof of the equivalence " $(i) \Leftrightarrow(i i)$ " is concluded. The last part is obvious.

Remark 2.2. We observe that, in the case when

$$
\lambda_{r}(t)=r[1-\exp (-t)], t \geq 0,
$$

then the function $f$ is $\lambda_{r}$-convex on convex subset $C$ of a linear space $X$ iff

$$
f\left(\frac{\alpha x+\beta y}{\alpha+\beta}\right) \leq \frac{[1-\exp (-\alpha)] f(x)+[1-\exp (-\beta)] f(y)}{1-\exp (-\alpha-\beta)}
$$

for any $\alpha, \beta \geq 0$ with $\alpha+\beta>0$ and $x, y \in C$.

We observe that this definition is independent of $r>0$.

The inequality (29) is equivalent to

$$
f\left(\frac{\alpha x+\beta y}{\alpha+\beta}\right) \leq \frac{\exp (\beta)[\exp (\alpha)-1] f(x)+\exp (\alpha)[\exp (\beta)-1] f(y)}{\exp (\alpha+\beta)-1}
$$

for any $\alpha, \beta \geq 0$ with $\alpha+\beta>0$ and $x, y \in C$. 


\section{Hermite-Hadamard Type Inequalities}

For an arbitrary mapping $f: C \subset X \rightarrow \mathbb{R}$ where $C$ is a convex subset of the linear space $X$, we can define the mapping

$$
g_{x, y}:[0,1] \rightarrow \mathbb{R}, g_{x, y}(t):=f(t x+(1-t) y),
$$

where $x, y$ are two distinct fixed elements in $C$.

Proposition 3.1. With the above assumptions, the following statements are equivalent:

(i) $f$ is $\lambda$-convex on $C$;

(ii) For every $x, y \in C$, the mapping $g_{x, y}$ is $\lambda$-convex on $[0,1]$.

Proof. " $(i) \Rightarrow($ ii $) "$. Let $t_{1}, t_{2} \in[0,1]$ and $\alpha, \beta \geq 0$ with $\alpha+\beta>0$. Then we have

$$
\begin{aligned}
& g_{x, y}\left(\frac{\alpha t_{1}+\beta t_{2}}{\alpha+\beta}\right) \\
& =f\left[\left(\frac{\alpha t_{1}+\beta t_{2}}{\alpha+\beta}\right) x+\left(1-\frac{\alpha t_{1}+\beta t_{2}}{\alpha+\beta}\right) y\right] \\
& =f\left[\frac{\alpha\left(t_{1} x+\left(1-t_{1}\right) y\right)+\beta\left(t_{2} x+\left(1-t_{2}\right) y\right)}{\alpha+\beta}\right] \\
& \leq \frac{\lambda(\alpha) f\left(t_{1} x+\left(1-t_{1}\right) y\right)+\lambda(\beta) f\left(t_{2} x+\left(1-t_{2}\right) y\right)}{\lambda(\alpha+\beta)} \\
& =\frac{\lambda(\alpha) g_{x, y}\left(t_{1}\right)+\lambda(\beta) g_{x, y}\left(t_{2}\right)}{\lambda(\alpha+\beta)}
\end{aligned}
$$

and the implication is proved.

$"($ ii $) \Rightarrow(i)$ ". Let $x, y \in C$ and $\alpha, \beta \geq 0$ with $\alpha+\beta>0$. Then we have

$$
\begin{aligned}
f\left(\frac{\alpha x+\beta y}{\alpha+\beta}\right) & =g_{x, y}\left(\frac{\alpha}{\alpha+\beta}\right)=g_{x, y}\left(\frac{\alpha \cdot 1+\beta \cdot 0}{\alpha+\beta}\right) \\
& \leq \frac{\lambda(\alpha) g_{x, y}(1)+\lambda(\beta) g_{x, y}(0)}{\lambda(\alpha+\beta)} \\
& =\frac{\lambda(\alpha) f(x)+\lambda(\beta) f(y)}{\lambda(\alpha+\beta)}
\end{aligned}
$$

and the implication is thus proved.

We can introduce the following mapping $k_{x, y}:[0,1] \rightarrow \mathbb{R}$

$$
k_{x, y}(t):=\frac{1}{2}[f(t x+(1-t) y)+f((1-t) x+t y)]
$$

for $x, y \in C, x \neq y$. 
Theorem 3.1. Let $f: C \rightarrow[0, \infty)$ be a $\lambda$-convex function on $C$. Assume that $x, y \in C$ with $x \neq y$.

(i) We have the equality

$$
k_{x, y}(1-t)=k_{x, y}(t) \text { for all } t \in[0,1] ;
$$

(ii) The mapping $k_{x, y}$ is $\lambda$-convex on $[0,1]$;

(iii) One has the inequalities

$$
k_{x, y}(t) \leq \frac{\lambda(t)+\lambda(1-t)}{\lambda(1)} \cdot \frac{f(x)+f(y)}{2}
$$

and

$$
\frac{\lambda(2 \alpha)}{2 \lambda(\alpha)} f\left(\frac{x+y}{2}\right) \leq k_{x, y}(t)
$$

for all $t \in[0,1]$ and $\alpha>0$.

(iv) Let $y, x \in C$ with $y \neq x$ and assume that the mappings $[0,1] \ni t \mapsto$ $f[(1-t) x+t y]$ and $\lambda$ are Lebesgue integrable on $[0,1]$, then we have the Hermite-Hadamard type inequalities

$$
\frac{\lambda(2 \alpha)}{2 \lambda(\alpha)} f\left(\frac{x+y}{2}\right) \leq \int_{0}^{1} f((1-t) x+t y) d t<\frac{f(x)+f(y)}{\lambda(1)} \int_{0}^{1} \lambda(t) d t
$$

for any $\alpha>0$.

Proof. The statements (i) and (ii) are obvious.

(iii). By the $\lambda$-convexity of $f$ we have:

$$
f(t x+(1-t) y) \leq \frac{\lambda(t) f(x)+\lambda(1-t) f(y)}{\lambda(1)}
$$

and

$$
f((1-t) x+t y) \leq \frac{\lambda(1-t) f(x)+\lambda(t) f(y)}{\lambda(1)},
$$

which gives by addition inequality (32).

We also have

$$
\frac{\lambda(\alpha) f(z)+\lambda(\alpha) f(u)}{\lambda(2 \alpha)} \geq f\left(\frac{\alpha z+\alpha u}{\alpha+\alpha}\right)=f\left(\frac{z+u}{2}\right)
$$

i.e.,

for all $z, u \in C$.

$$
\frac{\lambda(\alpha)}{\lambda(2 \alpha)}[f(z)+f(u)] \geq f\left(\frac{z+u}{2}\right)
$$

If we write this inequality for $z=t x+(1-t) y$ and $u=(1-t) x+t y$ we get

$$
\frac{\lambda(\alpha)}{\lambda(2 \alpha)}[f(t x+(1-t) y)+f((1-t) x+t y)] \geq f\left(\frac{x+y}{2}\right),
$$


which is equivalent to (33).

Integrating (33) and (34) over $t$ on $[0,1]$ we get

$$
\begin{aligned}
\frac{2 \lambda(\alpha)}{\lambda(2 \alpha)} \cdot f\left(\frac{x+y}{2}\right) & \leq \frac{1}{2} \int_{0}^{1}[f(t x+(1-t) y)+f((1-t) x+t y)] d t \\
& \leq \frac{f(x)+f(y)}{2} \int_{0}^{1} \frac{\lambda(t)+\lambda(1-t)}{\lambda(1)} d t
\end{aligned}
$$

Since

and

$$
\int_{0}^{1} f(t x+(1-t) y) d t=\int_{0}^{1} f((1-t) x+t y) d t
$$

$$
\int_{0}^{1} \lambda(t) d t=\int_{0}^{1} \lambda(1-t) d t
$$

then by (35) we get the desired result (34).

Remark 3.1. Since $\lambda$ is subadditive, then

$$
\frac{\lambda(2 \alpha)}{2 \lambda(\alpha)} \leq 1 \text { for any } \alpha>0
$$

From (34) we have the best inequality

$$
\begin{aligned}
\sup _{\alpha>0}\left\{\frac{\lambda(2 \alpha)}{2 \lambda(\alpha)}\right\} f\left(\frac{x+y}{2}\right) & \leq \int_{0}^{1} f((1-t) x+t y) d t \\
& \leq \frac{f(x)+f(y)}{\lambda(1)} \int_{0}^{1} \lambda(t) d t
\end{aligned}
$$

If the right limit

$$
k=\lim _{s \rightarrow 0+} \frac{\lambda(s)}{s}
$$

exists and is finite with $k>0$, then

$$
\lim _{\alpha \rightarrow 0+} \frac{\lambda(2 \alpha)}{2 \lambda(\alpha)}=\lim _{\alpha \rightarrow 0+} \frac{\left(\frac{\lambda(2 \alpha)}{2 \alpha}\right)}{\left(\frac{\lambda(\alpha)}{\alpha}\right)}=\frac{\lim _{\alpha \rightarrow 0+}\left(\frac{\lambda(2 \alpha)}{2 \alpha}\right)}{\lim _{\alpha \rightarrow 0+}\left(\frac{\lambda(\alpha)}{\alpha}\right)}=\frac{k}{k}=1
$$

and by (34) we get

$$
f\left(\frac{x+y}{2}\right) \leq \int_{0}^{1} f((1-t) x+t y) d t \leq \frac{f(x)+f(y)}{\lambda(1)} \int_{0}^{1} \lambda(t) d t .
$$

Corollary 3.1. Assume that the function $f: C \rightarrow[0, \infty)$ is $\lambda_{r}$-convex with $\lambda_{r}:[0, \infty) \rightarrow[0, \infty)$,

$$
\lambda_{r}(t):=\ln \left[\frac{h(r)}{h(r \exp (-t))}\right]
$$


and $h$ is as in Corollary 2.1.

If $y, x \in C$ with $y \neq x$ and the mapping $[0,1] \ni t \mapsto f[(1-t) x+t y]$ is Lebesgue integrable on $[0,1]$, then we have the Hermite-Hadamard type inequalities

$$
\begin{aligned}
f\left(\frac{x+y}{2}\right) & \leq \int_{0}^{1} f((1-t) x+t y) d t \\
& \leq \frac{f(x)+f(y)}{\ln \left[\frac{h(r)}{h\left(r e^{-1}\right)}\right]} \int_{0}^{1} \ln \left[\frac{h(r)}{h(r \exp (-t))}\right] d t .
\end{aligned}
$$

Proof. We know that $\lambda_{r}$ is differentiable on $(0, \infty)$ and

$$
\lambda_{r}^{\prime}(t):=\frac{r \exp (-t) h^{\prime}(r \exp (-t))}{h(r \exp (-t))}
$$

for $t \in(0, \infty)$, where

$$
h^{\prime}(z)=\sum_{n=1}^{\infty} n a_{n} z^{n-1}
$$

Since $\lambda_{r}(0)=0$, then

$$
k=\lim _{s \rightarrow 0+} \frac{\lambda(s)}{s}=\lambda_{+}^{\prime}(0)=\frac{r h^{\prime}(r)}{h(r)}>0 \text { for } r \in(0, R)
$$

and by (37) we get (38).

Furthermore, we observe that the following elementary inequality holds:

$$
(\alpha+\beta)^{p} \geq(\leq) \alpha^{p}+\beta^{p}
$$

for any $\alpha, \beta \geq 0$ and $p \geq 1(0<p<1)$.

Indeed, if we consider the function $f_{p}:[0, \infty) \rightarrow \mathbb{R}, f_{p}(t)=(t+1)^{p}-t^{p}$ we have $f_{p}^{\prime}(t)=p\left[(t+1)^{p-1}-t^{p-1}\right]$. Observe that for $p>1$ and $t>0$ we have that $f_{p}^{\prime}(t)>0$ showing that $f_{p}$ is strictly increasing on the interval $[0, \infty)$. Now for $t=\frac{\alpha}{\beta}(\beta>0, \alpha \geq 0)$ we have $f_{p}(t)>f_{p}(0)$ giving that $\left(\frac{\alpha}{\beta}+1\right)^{p}-\left(\frac{\alpha}{\beta}\right)^{p}>1$, i.e., the desired inequality (39).

For $p \in(0,1)$ we have $f_{p}$ strictly decreasing on $[0, \infty)$ which proves the second case in (39).

If we consider the power function $\hat{\lambda}_{q}(t)=t^{q}$ with $q \in(0,1)$, then $\hat{\lambda}_{q}$ is subadditive and by (34) we have

$$
\frac{1}{2^{1-q}} f\left(\frac{x+y}{2}\right) \leq \int_{0}^{1} f((1-t) x+t y) d t \leq \frac{f(x)+f(y)}{q+1},
$$

therefore we recapture the inequality (12) that was obtained from (7). 
For $q \geq 1$ and if we consider the function $\check{\lambda}_{q}(t)=\frac{1}{t^{q}}$, then for any $t, s>0$ we have

$$
\check{\lambda}_{q}(t+s)=\frac{1}{(t+s)^{q}} \leq \frac{1}{t^{s}+s^{q}} \leq \frac{1}{t^{s}}+\frac{1}{s^{q}}=\check{\lambda}_{q}(t)+\check{\lambda}_{q}(s)
$$

which shows that $\check{\lambda}_{q}$ is subadditive.

If $f: C \rightarrow[0, \infty)$ is a $\check{\lambda}_{q}$-convex function on $C$, i.e.

$$
f\left(\frac{\alpha x+\beta y}{\alpha+\beta}\right) \leq \frac{\alpha^{-q} f(x)+\beta^{-q} f(y)}{(\alpha+\beta)^{-q}}
$$

for all $\alpha, \beta \geq 0$ with $\alpha+\beta>0$ and $x, y \in C$, where $q \geq 1$, then we observe that the inequality (41) is equivalent to

$$
f\left(\frac{\alpha x+\beta y}{\alpha+\beta}\right) \leq\left(\frac{\alpha+\beta}{\alpha \beta}\right)^{q}\left[\beta^{q} f(x)+\alpha^{q} f(y)\right]
$$

for all $\alpha, \beta \geq 0$ with $\alpha+\beta>0$ and $x, y \in C$, where $q \geq 1$.

Since $\check{\lambda}_{q}$ is not integrable on $[0,1]$ we cannot apply the second inequality from (34). However, from the first inequality we get

$$
\frac{1}{2^{q+1}} f\left(\frac{x+y}{2}\right) \leq \int_{0}^{1} f((1-t) x+t y) d t
$$

provided that $f$ is $\check{\lambda}_{q}$-convex and the integral $\int_{0}^{1} f((1-t) x+t y) d t$ exists for some $x, y \in C$.

Moreover, if we assume that $f: C \rightarrow[0, \infty)$ is a $\lambda$-convex function on $C$ with $\lambda(t)=1-\exp (-t), t \geq 0$, i.e.

$$
f\left(\frac{\alpha x+\beta y}{\alpha+\beta}\right) \leq \frac{\exp (\beta)[\exp (\alpha)-1] f(x)+\exp (\alpha)[\exp (\beta)-1] f(y)}{\exp (\alpha+\beta)-1}
$$

for any $\alpha, \beta \geq 0$ with $\alpha+\beta>0$ and $x, y \in C$, then by (37) we have

$f\left(\frac{x+y}{2}\right) \leq \int_{0}^{1} f((1-t) x+t y) d t \leq \frac{f(x)+f(y)}{1-e^{-1}} \int_{0}^{1}[1-\exp (-t)] d t$,

that is equivalent to

$$
f\left(\frac{x+y}{2}\right) \leq \int_{0}^{1} f((1-t) x+t y) d t \leq \frac{f(x)+f(y)}{e-1},
$$

provided the integral $\int_{0}^{1} f((1-t) x+t y) d t$ exists for some $x, y \in C$.

\section{Inequalities for Double Integrals}

We have the following result: 
Theorem 4.1. Let $f: C \rightarrow[0, \infty)$ be a $\lambda$-convex function on $C$. Let $y, x \in C$ with $y \neq x$ and assume that the mappings $[0,1] \ni t \mapsto f[(1-t) x+t y]$ and $\lambda$ are Lebesgue integrable on $[0,1]$, then for $0 \leq a<b$ we have the Hermite-Hadamard type inequalities

$$
\begin{aligned}
& \frac{\lambda(2 \eta)}{2 \lambda(\eta)} f\left(\frac{x+y}{2}\right)(b-a)^{2} \\
& \leq \frac{1}{2} \int_{a}^{b} \int_{a}^{b}\left[f\left(\frac{\alpha x+\beta y}{\alpha+\beta}\right) d \alpha d \beta+f\left(\frac{\beta x+\alpha y}{\alpha+\beta}\right)\right] d \alpha d \beta \\
& \leq[f(x)+f(y)] \int_{a}^{b} \int_{a}^{b} \frac{\lambda(\alpha)}{\lambda(\alpha+\beta)} d \alpha d \beta
\end{aligned}
$$

for any $\eta>0$.

Proof. By the $\lambda$-convexity of $f$ we have

$$
f\left(\frac{\alpha x+\beta y}{\alpha+\beta}\right) \leq \frac{\lambda(\alpha) f(x)+\lambda(\beta) f(y)}{\lambda(\alpha+\beta)}
$$

and

$$
f\left(\frac{\beta x+\alpha y}{\alpha+\beta}\right) \leq \frac{\lambda(\beta) f(x)+\lambda(\alpha) f(y)}{\lambda(\alpha+\beta)}
$$

for all $\alpha, \beta \geq 0$ with $\alpha+\beta>0$.

By adding these inequalities we obtain

$$
f\left(\frac{\alpha x+\beta y}{\alpha+\beta}\right)+f\left(\frac{\beta x+\alpha y}{\alpha+\beta}\right) \leq \frac{\lambda(\alpha)+\lambda(\beta)}{\lambda(\alpha+\beta)}[f(x)+f(y)]
$$

for all $\alpha, \beta \geq 0$ with $\alpha+\beta>0$.

Since the mappings $[0,1] \ni t \mapsto f[(1-t) x+t y]$ and $\lambda$ are Lebesgue integrable on $[0,1]$, then the integrals

$$
\int_{a}^{b} \int_{a}^{b} f\left(\frac{\alpha x+\beta y}{\alpha+\beta}\right) d \alpha d \beta \text { and } \int_{a}^{b} \int_{a}^{b} f\left(\frac{\beta x+\alpha y}{\alpha+\beta}\right) d \alpha d \beta
$$

exist and by integrating the inequality (47) on the square $[a, b]^{2}$ we get

$$
\begin{aligned}
& \int_{a}^{b} \int_{a}^{b} f\left(\frac{\alpha x+\beta y}{\alpha+\beta}\right) d \alpha d \beta+\int_{a}^{b} \int_{a}^{b} f\left(\frac{\beta x+\alpha y}{\alpha+\beta}\right) d \alpha d \beta \\
& \leq[f(x)+f(y)] \int_{a}^{b} \int_{a}^{b} \frac{\lambda(\alpha)+\lambda(\beta)}{\lambda(\alpha+\beta)} d \alpha d \beta \\
& =2[f(x)+f(y)] \int_{a}^{b} \int_{a}^{b} \frac{\lambda(\alpha)}{\lambda(\alpha+\beta)} d \alpha d \beta
\end{aligned}
$$

and the second inequality in (46) is proved. 
We know from the proof of Theorem 3.1 that

$$
\frac{\lambda(\eta)}{\lambda(2 \eta)}[f(z)+f(u)] \geq f\left(\frac{z+u}{2}\right)
$$

for all $z, u \in C$ and $\eta>0$.

Taking

$$
z=\frac{\alpha x+\beta y}{\alpha+\beta} \text { and } u=\frac{\beta x+\alpha y}{\alpha+\beta}
$$

we get

$$
\frac{\lambda(\eta)}{\lambda(2 \eta)}\left[f\left(\frac{\alpha x+\beta y}{\alpha+\beta}\right)+f\left(\frac{\beta x+\alpha y}{\alpha+\beta}\right)\right] \geq f\left(\frac{x+y}{2}\right)
$$

for all $\alpha, \beta \geq 0$ with $\alpha+\beta>0$ and $\eta>0$.

Integrating inequality (48) on the square $[a, b]^{2}$ we get the first part of (46).

Remark 4.1. If we write inequality (46) for $f: C \rightarrow[0, \infty)$ a $\check{\lambda}_{q}$-convex function on $C$, then we get the inequality

$$
\begin{aligned}
& \frac{1}{2^{q+1}} f\left(\frac{x+y}{2}\right)(b-a)^{2} \\
& \leq \frac{1}{2} \int_{a}^{b} \int_{a}^{b}\left[f\left(\frac{\alpha x+\beta y}{\alpha+\beta}\right) d \alpha d \beta+f\left(\frac{\beta x+\alpha y}{\alpha+\beta}\right)\right] d \alpha d \beta \\
& \leq[f(x)+f(y)] \int_{a}^{b} \int_{a}^{b}\left(\frac{\alpha+\beta}{\alpha}\right)^{q} d \alpha d \beta,
\end{aligned}
$$

provided that the mapping $[0,1] \ni t \mapsto f[(1-t) x+t y]$ is Lebesgue integrable on $[0,1]$.

For $q=1$ we have

$$
\begin{aligned}
\int_{a}^{b} \int_{a}^{b} \frac{\alpha+\beta}{\alpha} d \beta d \alpha & =\int_{a}^{b} \int_{a}^{b}\left(1+\frac{\beta}{\alpha}\right) d \beta d \alpha \\
& =(b-a)^{2}+(\ln b-\ln a) \frac{b^{2}-a^{2}}{2} \\
& =(b-a)^{2}\left(1+\frac{\ln b-\ln a}{b-a} \cdot \frac{a+b}{2}\right) \\
& =(b-a)^{2}\left[1+\frac{A(a, b)}{L(a, b)}\right]
\end{aligned}
$$

where

$$
L(a, b):=\frac{b-a}{\ln b-\ln a}
$$

is the logarithmic mean. 
Then from (49) we get

$$
\begin{aligned}
& \frac{1}{4} f\left(\frac{x+y}{2}\right) \\
& \leq \frac{1}{2(b-a)^{2}} \int_{a}^{b} \int_{a}^{b}\left[f\left(\frac{\alpha x+\beta y}{\alpha+\beta}\right) d \alpha d \beta+f\left(\frac{\beta x+\alpha y}{\alpha+\beta}\right)\right] d \alpha d \beta \\
& \leq[f(x)+f(y)]\left[1+\frac{A(a, b)}{L(a, b)}\right]
\end{aligned}
$$

provided that $f: C \rightarrow[0, \infty)$ is a $\check{\lambda}_{1}$-convex function on $C$ and the mapping $[0,1] \ni t \mapsto f[(1-t) x+t y]$ is Lebesgue integrable on $[0,1]$.

For $q=2$ we have

$$
\begin{aligned}
\int_{a}^{b} \int_{a}^{b}\left(\frac{\alpha+\beta}{\alpha}\right)^{2} d \beta d \alpha & =\int_{a}^{b} \int_{a}^{b}\left(1+\frac{\beta}{\alpha}\right)^{2} d \beta d \alpha \\
& =\int_{a}^{b} \int_{a}^{b}\left(1+\frac{2 \beta}{\alpha}+\frac{\beta^{2}}{\alpha^{2}}\right) d \beta d \alpha \\
& =(b-a)^{2}\left(1+2 \frac{\ln b-\ln a}{b-a} \cdot \frac{a+b}{2}+\frac{a^{2}+a b+b^{2}}{3 a b}\right) \\
& =\left(2 \frac{\ln b-\ln a}{b-a} \cdot \frac{a+b}{2}+\frac{a^{2}+4 a b+b^{2}}{3 a b}\right) \\
& =2(b-a)^{2}\left[\frac{1}{3}+\frac{2}{3} \cdot \frac{A(a, b)}{G(a, b)}+\frac{A(a, b)}{L(a, b)}\right]
\end{aligned}
$$

where $G(a, b):=\sqrt{a b}$ is the geometric mean.

Then from (49) we get

$$
\begin{aligned}
& \frac{1}{8} f\left(\frac{x+y}{2}\right) \\
& \leq \frac{1}{2(b-a)^{2}} \int_{a}^{b} \int_{a}^{b}\left[f\left(\frac{\alpha x+\beta y}{\alpha+\beta}\right) d \alpha d \beta+f\left(\frac{\beta x+\alpha y}{\alpha+\beta}\right)\right] d \alpha d \beta \\
& \leq 2[f(x)+f(y)]\left[\frac{1}{3}+\frac{2}{3} \cdot \frac{A(a, b)}{G(a, b)}+\frac{A(a, b)}{L(a, b)}\right]
\end{aligned}
$$

provided that $f: C \rightarrow[0, \infty)$ is a $\check{\lambda}_{2}$-convex function on $C$ and the mapping $[0,1] \ni t \mapsto f[(1-t) x+t y]$ is Lebesgue integrable on $[0,1]$.

Open Access: This article is distributed under the terms of the Creative Commons Attribution License (CC-BY 4.0) which permits any use, distribution, and reproduction in any medium, provided the original author(s) and the source are credited. 


\section{References}

[1] M. Alomari and M. Darus, The Hadamard's inequality for s-convex function. Int. J. Math. Anal. (Ruse) 2 (2008), no. 13-16, 639-646.

[2] M. Alomari and M. Darus, Hadamard-type inequalities for s-convex functions. Int. Math. Forum 3 (2008), no. 37-40, 1965-1975.

[3] G. A. Anastassiou, Univariate Ostrowski inequalities, revisited. Monatsh. Math., 135 (2002), no. 3, 175-189.

[4] N. S. Barnett, P. Cerone, S. S. Dragomir, M. R. Pinheiro,and A. Sofo, Ostrowski type inequalities for functions whose modulus of the derivatives are convex and applications. Inequality Theory and Applications, Vol. 2 (Chinju/Masan, 2001), 19-32, Nova Sci. Publ., Hauppauge, NY, 2003. Preprint: RGMIA Res. Rep. Coll. 5 (2002), No. 2, Art. 1 [Online http://rgmia.org/papers/v5n2/Paperwapp2q.pdf].

[5] E. F. Beckenbach, Convex functions, Bull. Amer. Math. Soc. 54(1948), 439-460.

[6] M. Bombardelli and S. Varošanec, Properties of h-convex functions related to the Hermite-Hadamard-Fejér inequalities. Comput. Math. Appl. 58 (2009), no. 9, 1869-1877.

[7] W. W. Breckner, Stetigkeitsaussagen für eine Klasse verallgemeinerter konvexer Funktionen in topologischen linearen Räumen. (German) Publ. Inst. Math. (Beograd) (N.S.) 23(37) (1978), 13-20.

[8] W. W. Breckner and G. Orbán, Continuity properties of rationally s-convex mappings with values in an ordered topological linear space. Universitatea "BabeşBolyai", Facultatea de Matematica, Cluj-Napoca, 1978. viii+92 pp.

[9] P. Cerone and S. S. Dragomir, Midpoint-type rules from an inequalities point of view, Ed. G. A. Anastassiou, Handbook of Analytic-Computational Methods in Applied Mathematics, CRC Press, New York. 135-200.

[10] P. Cerone and S. S. Dragomir, New bounds for the three-point rule involving the Riemann-Stieltjes integrals, in Advances in Statistics Combinatorics and Related Areas, C. Gulati, et al. (Eds.), World Science Publishing, 2002, 53-62.

[11] P. Cerone, S. S. Dragomir and J. Roumeliotis, Some Ostrowski type inequalities for $n$-time differentiable mappings and applications, Demonstratio Mathematica, 32(2) (1999), 697-712.

[12] G. Cristescu, Hadamard type inequalities for convolution of $h$-convex functions. Ann. Tiberiu Popoviciu Semin. Funct. Equ. Approx. Convexity 8 (2010), 3-11.

[13] S. S. Dragomir, Ostrowski's inequality for monotonous mappings and applications, J. KSIAM, 3(1) (1999), 127-135.

[14] S. S. Dragomir, The Ostrowski's integral inequality for Lipschitzian mappings and applications, Comp. Math. Appl., 38 (1999), 33-37.

[15] S. S. Dragomir, On the Ostrowski's inequality for Riemann-Stieltjes integral, Korean J. Appl. Math., 7 (2000), 477-485.

[16] S. S. Dragomir, On the Ostrowski's inequality for mappings of bounded variation and applications, Math. Ineq. \& Appl., 4(1) (2001), 33-40.

[17] S. S. Dragomir, On the Ostrowski inequality for Riemann-Stieltjes integral $\int_{a}^{b} f(t) d u(t)$ where $f$ is of Hölder type and $u$ is of bounded variation and applications, J. KSIAM, 5(1) (2001), 35-45.

[18] S. S. Dragomir, Ostrowski type inequalities for isotonic linear functionals, $J$. Inequal. Pure \& Appl. Math., 3(5) (2002), Art. 68. 
[19] S. S. Dragomir, An inequality improving the first Hermite-Hadamard inequality for convex functions defined on linear spaces and applications for semi-inner products. J. Inequal. Pure Appl. Math. 3 (2002), no. 2, Article 31, 8 pp.

[20] S. S. Dragomir, An inequality improving the first Hermite-Hadamard inequality for convex functions defined on linear spaces and applications for semi-inner products, J. Inequal. Pure Appl. Math. 3 (2002), No. 2, Article 31.

[21] S. S. Dragomir, An inequality improving the second Hermite-Hadamard inequality for convex functions defined on linear spaces and applications for semi-inner products, J. Inequal. Pure Appl. Math. 3 (2002), No.3, Article 35.

[22] S. S. Dragomir, An Ostrowski like inequality for convex functions and applications, Revista Math. Complutense, 16(2) (2003), 373-382.

[23] S. S. Dragomir, Operator Inequalities of Ostrowski and Trapezoidal Type. Springer Briefs in Mathematics. Springer, New York, 2012. x+112 pp. ISBN: 978-1-46141778-1

[24] S. S. Dragomir, Bounds for the normalised Jensen functional, Bull. Austral. Math. Soc. 74 (2006), pp. 471-478.

[25] S. S. Dragomir, P. Cerone, J. Roumeliotis and S. Wang, A weighted version of Ostrowski inequality for mappings of Hölder type and applications in numerical analysis, Bull. Math. Soc. Sci. Math. Romanie, 42(90) (4) (1999), 301-314.

[26] S. S. Dragomir and S. Fitzpatrick, The Hadamard inequalities for s-convex functions in the second sense. Demonstratio Math. 32 (1999), no. 4, 687-696.

[27] S. S. Dragomir and S. Fitzpatrick, The Jensen inequality for s-Breckner convex functions in linear spaces. Demonstratio Math. 33 (2000), no. 1, 43-49.

[28] S. S. Dragomir and B. Mond, On Hadamard's inequality for a class of functions of Godunova and Levin. Indian J. Math. 39 (1997), no. 1, 1-9.

[29] S. S. Dragomir and C. E. M. Pearce, On Jensen's inequality for a class of functions of Godunova and Levin. Period. Math. Hungar. 33 (1996), no. 2, 93-100.

[30] S. S. Dragomir and C. E. M. Pearce, Quasi-convex functions and Hadamard's inequality, Bull. Austral. Math. Soc. 57 (1998), 377-385.

[31] S. S. Dragomir, J. Pečarić and L. Persson, Some inequalities of Hadamard type. Soochow J. Math. 21 (1995), no. 3, 335-341.

[32] S. S. Dragomir, J. Pečarić and L. Persson, Properties of some functionals related to Jensen's inequality, Acta Math. Hungarica, 70 (1996), 129-143.

[33] S. S. Dragomir and Th. M. Rassias (Eds), Ostrowski Type Inequalities and Applications in Numerical Integration, Kluwer Academic Publisher, 2002.

[34] S. S. Dragomir and S. Wang, A new inequality of Ostrowski's type in $L_{1}-$ norm and applications to some special means and to some numerical quadrature rules, Tamkang J. of Math., 28 (1997), 239-244.

[35] S. S. Dragomir and S. Wang, Applications of Ostrowski's inequality to the estimation of error bounds for some special means and some numerical quadrature rules, Appl. Math. Lett., 11 (1998), 105-109.

[36] S. S. Dragomir and S. Wang, A new inequality of Ostrowski's type in $L_{p}-$ norm and applications to some special means and to some numerical quadrature rules, Indian J. of Math., 40(3) (1998), 245-304.

[37] A. El Farissi, Simple proof and refinement of Hermite-Hadamard inequality, $J$. Math. Ineq. 4 (2010), No. 3, 365-369. 
[38] E. K. Godunova and V. I. Levin, Inequalities for functions of a broad class that contains convex, monotone and some other forms of functions. (Russian) Numerical mathematics and mathematical physics (Russian), 138-142, 166, Moskov. Gos. Ped. Inst., Moscow, 1985

[39] H. Hudzik and L. Maligranda, Some remarks on s-convex functions. Aequationes Math. 48 (1994), no. 1, 100-111.

[40] E. Kikianty and S. S. Dragomir, Hermite-Hadamard's inequality and the p-HHnorm on the Cartesian product of two copies of a normed space, Math. Inequal. Appl. (in press)

[41] U. S. Kirmaci, M. Klaričić Bakula, M. E Özdemir and J. Pečarić, Hadamardtype inequalities for s-convex functions. Appl. Math. Comput. 193 (2007), no. 1, $26-35$.

[42] M. A. Latif, On some inequalities for h-convex functions. Int. J. Math. Anal. (Ruse) 4 (2010), no. 29-32, 1473-1482.

[43] D. S. Mitrinović and I. B. Lacković, Hermite and convexity, Aequationes Math. 28 (1985), 229-232.

[44] D. S. Mitrinović and J. E. Pečarić, Note on a class of functions of Godunova and Levin. C. R. Math. Rep. Acad. Sci. Canada 12 (1990), no. 1, 33-36.

[45] C. E. M. Pearce and A. M. Rubinov, P-functions, quasi-convex functions, and Hadamard-type inequalities. J. Math. Anal. Appl. 240 (1999), no. 1, 92-104.

[46] J. E. Pečarić and S. S. Dragomir, On an inequality of Godunova-Levin and some refinements of Jensen integral inequality. Itinerant Seminar on Functional Equations, Approximation and Convexity (Cluj-Napoca, 1989), 263-268, Preprint, 89-6, Univ. "Babeş-Bolyai", Cluj-Napoca, 1989.

[47] J. Pečarić and S. S. Dragomir, A generalization of Hadamard's inequality for isotonic linear functionals, Radovi Mat. (Sarajevo) 7 (1991), 103-107.

[48] M. Radulescu, S. Radulescu and P. Alexandrescu, On the Godunova-Levin-Schur class of functions. Math. Inequal. Appl. 12 (2009), no. 4, 853-862.

[49] M. Z. Sarikaya, A. Saglam, and H. Yildirim, On some Hadamard-type inequalities for h-convex functions. J. Math. Inequal. 2 (2008), no. 3, 335-341.

[50] E. Set, M. E. Özdemir and M. Z. Sarıkaya, New inequalities of Ostrowski's type for s-convex functions in the second sense with applications. Facta Univ. Ser. Math. Inform. 27 (2012), no. 1, 67-82.

[51] M. Z. Sarikaya, E. Set and M. E. Özdemir, On some new inequalities of Hadamard type involving h-convex functions. Acta Math. Univ. Comenian. (N.S.) 79 (2010), no. $2,265-272$.

[52] M. Tunç, Ostrowski-type inequalities via h-convex functions with applications to special means. J. Inequal. Appl. 2013, 2013:326.

[53] S. Varošanec, On h-convexity. J. Math. Anal. Appl. 326 (2007), no. 1, 303-311. 